\title{
Synthesis and Characterization of Bimetallic CuNi Nanoparticles
}

\author{
P.Moganavally $^{1}$, R.Suresh ${ }^{2}$, M.Deepa ${ }^{3}$ \\ ${ }^{1 \& 2}$ (Research and Development Centre, Bharathiyar University, Coimbatore, India) \\ ${ }^{3}$ (Department of Chemistry, Muthurangam Govt. Arts College, Vellore, India)
}

\begin{abstract}
Bimetallic CuNi nanoparticles (BMN) have been successfully synthesized using metal chloride precursors via chemical reduction method without any protective agent and inert gas protection. The powder $X R D$ indicates the formation of CuNi nanoparticles is face-centered cubic structure (fcc) and the particle size as approximately $4 \mathrm{~nm}$ which is confirmed by finding the morphology using SEM analysis. The elemental composition reveals the formation of bimetallic alloy is in the ratio (1:1). The VSM data indicates the formed CuNi alloy exhibits the ferromagnetic behavior.
\end{abstract}

Keywords: Bimetallic nanoparticles (BMN), Chemical reduction, $x$-ray diffraction (XRD)

\section{Introduction}

In recent years, researchers focused on synthesizing metal nanoparticles, due to their unique electronic, optical, mechanical, magnetic and chemical properties. Several investigations indicates, when a metal is combined with another metal in bimetallic or alloy form, the properties of the resulting material could be enhanced with that of pure metals, due to their small size and large surface area. In case for Ni-based bimetallic nanoparticles containing copper exhibit better selectivity [1] and catalytic activity [2] than monometallic nickel.

Various physical and chemical routes, such as template growth [3-5], organometallic-precursor thermolysis [6], solution chemical reduction [7], sol-gel processing [8] and chemical vapour deposition [9, 10] were applied to prepare metal nanoparticles. The chemical reduction method is a good way to obtain amorphous alloy nanoparticles.

In this paper, we present results on the synthesis of $\mathrm{CuNi}$ bimetallic nanoparticles using hydrazine reduction method in the absence of polymer or any other extra protective agent, since ethylene glycol with hydrazine hydrate acts as protective agent as well as reducing agent.

\subsection{Chemicals}

\section{Experiment}

All the chemicals of reagent grade quality were used without further purification; including Copper chloride hydrate $\left(\mathrm{CuCl}_{2} \cdot 2 \mathrm{H}_{2} \mathrm{O}\right)$, Nickel Chloride hexahydate $\left(\mathrm{NiCl}_{2} \cdot 6 \mathrm{H}_{2} \mathrm{O}\right)$, Sodium hydroxide $(\mathrm{NaOH})$, Ethylene glycol $\left(\mathrm{C}_{2} \mathrm{H}_{6} \mathrm{O}_{2}\right)$ and Hydrazine hydrate $\left(\mathrm{N}_{2} \mathrm{H}_{4} \cdot \mathrm{H}_{2} \mathrm{O}\right)$.

\subsection{Synthesis}

$\mathrm{CuNi}$ bimetallic nanoparticles were prepared by hydrazine reduction method using ethylene glycol as reported earlier [11-13]. An appropriate amount of metal chlorides in the ratio (1:1) was dissolved in universal solvent followed by addition of $3 \mathrm{ml}$ of hydrazine hydrate, ethylene glycol and required amount of sodium hydroxide $(1 \mathrm{M})$ was added with continuous stirring by maintaining the $\mathrm{pH}$ around 11.5 at $55 \square \mathrm{C}$.

$\mathrm{CuNi}$ nanoparticles were formed upon contact between precursor containing droplets and the hydrazine containing droplets. The reduction reactions could be expressed as

$$
\begin{aligned}
& 2 \mathrm{Ni}^{2+}+\mathrm{N}_{2} \mathrm{H}_{4}+4 \mathrm{OH}^{-} \rightarrow 2 \mathrm{Ni}+\mathrm{N}_{2}+4 \mathrm{H}_{2} \mathrm{O} \\
& 2 \mathrm{Cu}^{2+}+\mathrm{N}_{2} \mathrm{H}_{4}+4 \mathrm{OH}^{-} \rightarrow 2 \mathrm{Cu}+\mathrm{N}_{2}+4 \mathrm{H}_{2} \mathrm{O}
\end{aligned} \quad \text { and }
$$

The formed nanoparticles were collected, washed many times with acetone, and filtered dried at room temperature.

\subsection{Characterization}

The average crystallite size of the nanopowders were analyzed using powder $\mathrm{x}$-ray diffractometer using $\mathrm{CuK} \alpha(\lambda=1.5418 \AA)$ radiation. The morphology and structure of the sample was characterized by scanning electron microscopy (SEM). The elemental composition of bimetallic nanoparticles was determined from energy dispersive $\mathrm{x}$-ray spectra (EDS). 


\section{Results And Discussion}

The XRD patterns of CuNi bimetallic nanopowders are shown in "Fig.1". The lattice constant of the CuNi Nanoparticles was obtained from the peak angular positions $(2 \theta)$ and the corresponding miller indices (hkl) using Bragg's law,

$$
\mathrm{d}_{\mathrm{hkl}}=\mathrm{n} \lambda / 2 \sin \theta
$$

Where $\lambda$ is XRD wavelength, $\theta$ is peak angle for (hkl) each reflections.

The $2 \theta$ reflections at $43.6^{\circ}, 50.79^{\circ}$, and $70.37^{\circ}$ are identified as (111), (200) and (220) planes of CuNi nanoparticles and indexed to face cubic crystal structure. There were no observable lines in the XRD spectra corresponding to the pure $\mathrm{Cu}$ and $\mathrm{Ni}$. If the homogeneous solid solution of $\mathrm{CuNi}$ was not formed, the XRD spectra of pure $\mathrm{Cu}$ or $\mathrm{Ni}$ would be formed at the same time in the curve.

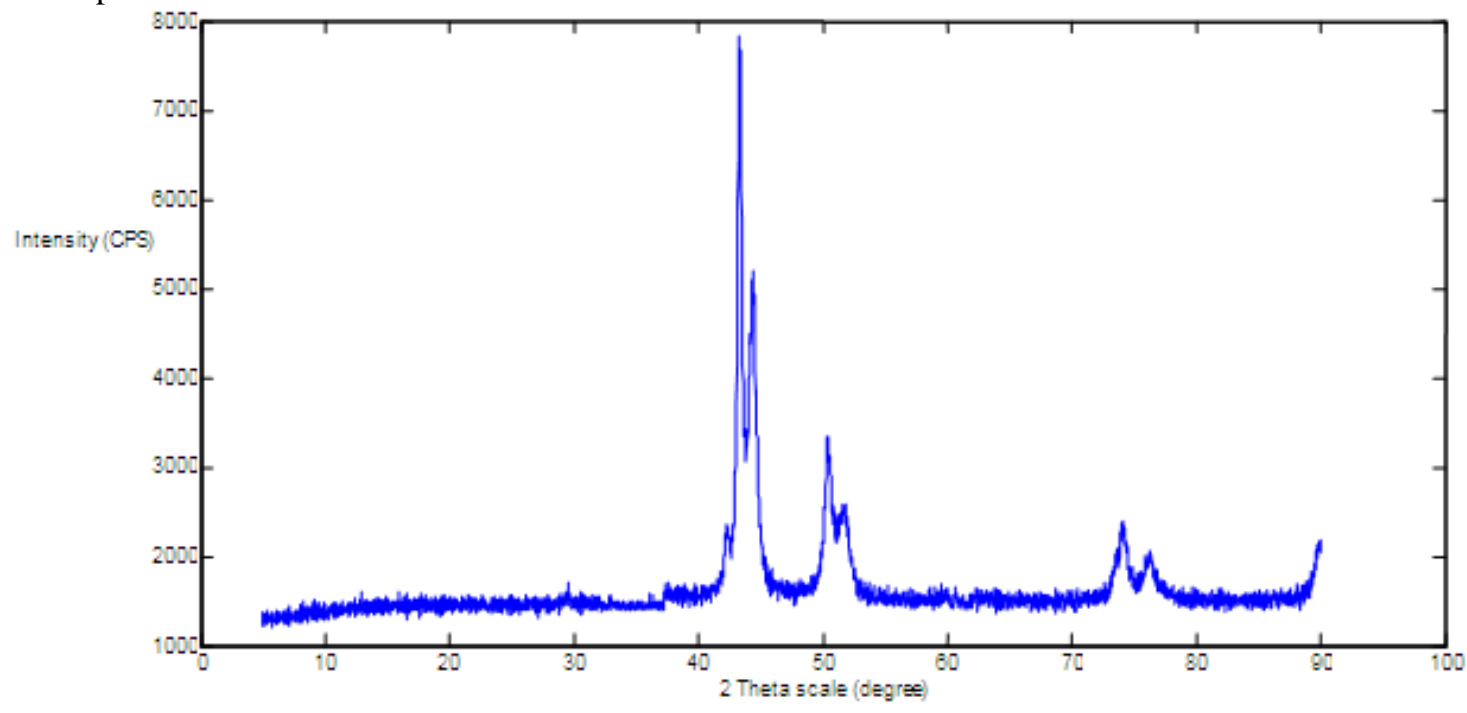

Figure 1 XRD pattern of CuNi nanoparticles

The average crystallite size was calculated using Debye-Scherer formula, and the obtained results are listed in "Table-1".

$$
\mathrm{D}=0.98 \lambda / \beta \operatorname{Cos} \theta
$$

Where $\mathrm{D}$ is the average crystallite site, $\beta$ is the broadening of FWHM of the intense peak, $\theta$ is the Bragg angle and $\lambda$ is radiation wavelength.

Table 1 XRD Parameters of CuNi BMN

\begin{tabular}{|l|l|l|l|l|}
\hline $\mathbf{2} \theta$ & $\theta$ & $\mathbf{D}$ & $\mathbf{d}(\mathbf{A})$ & $\mathbf{a}(\stackrel{\mathbf{A}}{)}$ \\
\hline 43.6066 & 21.8033 & 1.1088 & 2.0745 & 3.5932 \\
\hline 50.7933 & 25.395 & 2.833 & 1.7966 & 3.5933 \\
\hline 70.3733 & 35.185 & 3.1145 & 1.3372 & 3.7822 \\
\hline
\end{tabular}

The lattice constant ' $\mathrm{a}$ ' for cubic crystal system is calculated using

$$
\mathrm{a}=\mathrm{d}_{\mathrm{hkl}} \sqrt{ }\left(\mathrm{h}^{2}+\mathrm{k}^{2}+\mathrm{l}^{2}\right)
$$

The $d(\AA)$ value of $\mathrm{CuNi}$ bimetallic nanoparticles is found to be 2.0875 which is very close to that of given by JCPDS file PDF (09-0205).

"Fig.2" shows the SEM image of CuNi alloy nanoparticles is generally spherical with average size of $4.66 \mathrm{~nm}$.

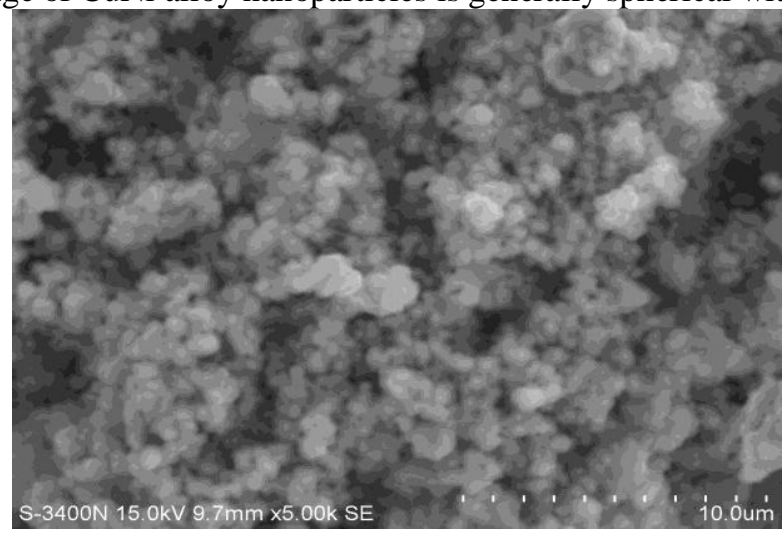

Figure 2 SEM image of CuNi nanoparticle 
"Fig.3" shows the EDS analysis of CuNi bimetallic nanoparticles which confirms the ratio (1:1) whose wt $\%$ of $\mathrm{Cu}$ and $\mathrm{Ni}$ is found to be 47.26 and 46.21. This also proves that $\mathrm{Cu}$ (II) and Ni (II) salts are almost reduced to their zero-valent metals.

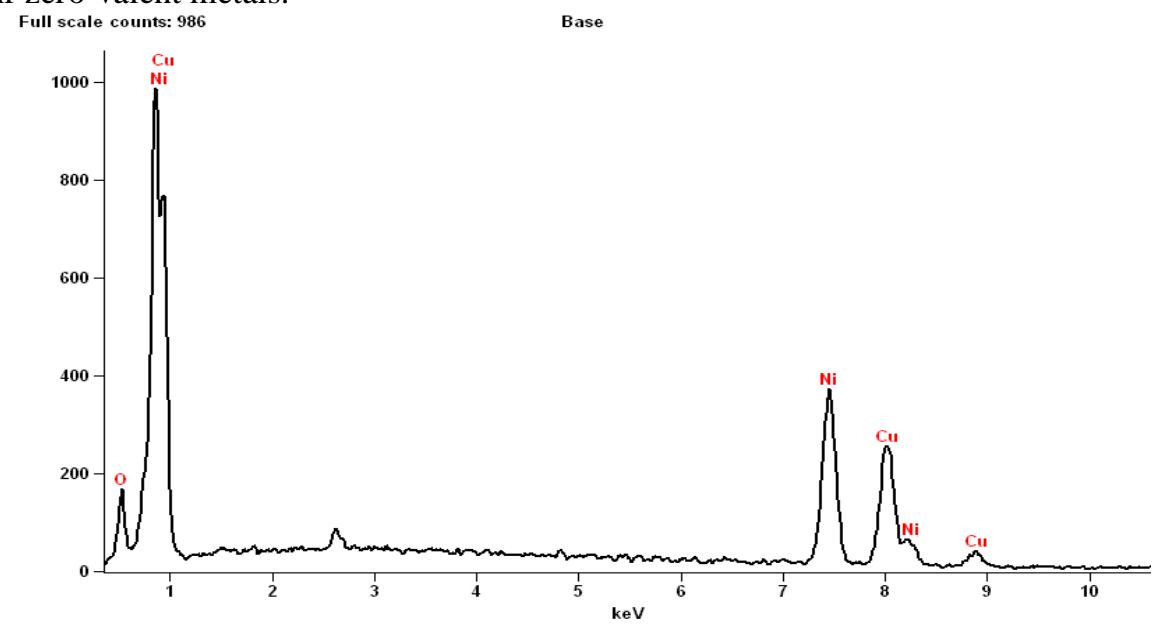

Figure 3 EDS image of CuNi Nanoparticles

The room temperature magnetization curve was measured using Vibrating Sample magnetometer."Fig.4" shows that the $\mathrm{M}-\mathrm{H}$ hysteresis loop of $\mathrm{CuNi}$ bimetallic nanoparticles reveals a ferromagnetic behavior with saturation magnetization (Ms), Coercitivity (Hc) is found to be $28.137 \times 10^{-3} \mathrm{emu}$, 12.63 KOe respectively.

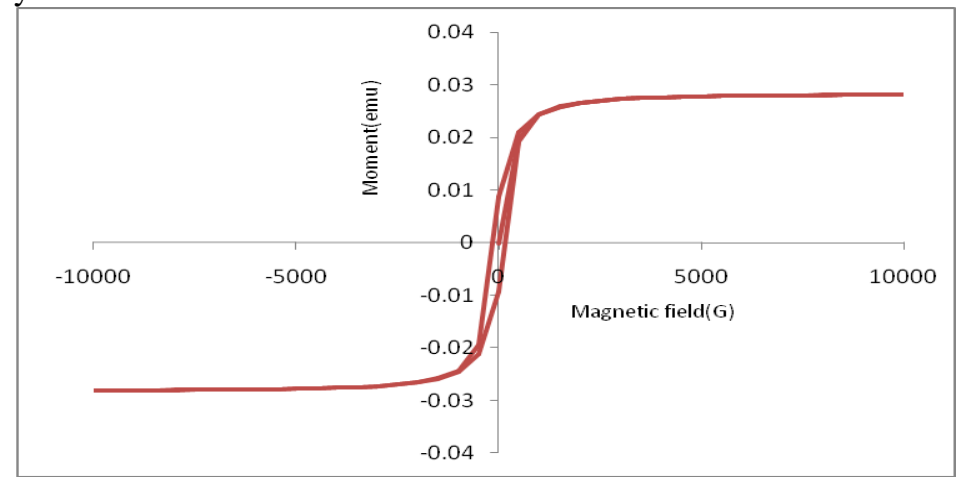

Figure 4 M-H Curves of CuNi nanoparticles

\section{Conclusion}

The CuNi bimetallic nanoparticles have been successfully synthesized at room temperature without using any protective agent and inert atmosphere. The XRD spectrum predicts CuNi BMNs is face centered cubic structure. We synthesized the $4.6 \mathrm{~nm}$ of particle size nanoparticles which shows the ferromagnetic behavior and it is confirmed through VSM spectrum. The method we synthesized is very simple, low cost, easy to operate and relatively cheap and eco-friendly.

\section{References}

[1]. F.M. Bautista, J.M. Campelo, A. Garca, R.Guarden, O.D. Luna and M.S. Sakanyan, J.Mol.Catal.: Chem., 104, 1996, $229-235$.

[2]. Y.Li, J.Chen, L.Chang and Y.Qin, J.Catal,,. 178, 1998, 76-83.

[3]. D.J. Pena, J.K.N.Mbindyo, A.J. Carado, T.E. Mallouk, D.K. Christine, B.Razavi, and T. S.Mayer, J.phys.Chem.B , 106, 30, 2002, 74587462.

[4]. W.C. Lin, S.S. Wong, P.C. Huang, C.B. Wu, B.R. Xu, C.T. Chang, H.Y. Yen and M.T. Lin, Appl. Phys.Lett., 89, 15, 2006, 153111/1 $153111 / 3$.

[5]. P.S. Alegaonkar, H.C. Lee, S.H. Lee, A.F. Moses, D. Fink and J.B. Yoo, J.Phys. D; Appl. Phys., 40,11, 2007, 3423-3429.

[6]. J. Gazquez, F. Sandiumenge, M. Coll, A.Pomar, N.Mesttres, T.Puig, X. Obradors, Y. Kihn, M.J. Casanove and C. Ballesteros, Chem.Mater., 18, 26, 2006, 6211-6219.

[7]. C.W. Kim, Y.H. Kim, H.G. Cha, H.W.Kwon and Y.S. Kang, J.Phys.Chem.B, 110, 48, 2006, 24418-24423.

[8]. C.P. Liu, M.W. Li, Z.Cui, J.R. Huang, Y.L. Tian, T. Lin and W.B. Mi, J.Mater.Sci., 42, 15, 2007, 6133-6138

[9]. S.J. Park, S. Kim, S.Lee, Z.G. Khim, K. Char and T. Hyeon, J.Am.Chem.Soc., 122, 2000, 8581-8587

[10]. N. Cordente, M. Respaud, F. Senocq, M.J. Casanove, C. Amiens and B. Chaudret, Nano.Lett. 1, 2001, 565-570.

[11]. Szu-Han Wu and Dong-Hwang Chen, J. Colloid Interrface Sci. 2003,259, 282

[12]. P. Saravanan, T.A. Jose, P.J. Thomas and G.U. Kulkarni, Bull. Mater. Sci..,2001, 24,515.

[13]. F. Fievet, J.P. Lagier and M. Figlarz, Sol. State Ionics, 1995, 82, 53. 